\title{
Relative and Interraction Effects of Errors in Physics Practical
}

\author{
Owolabi Olabode Thomas \\ Faculty of Education, Ekiti State University, Ado-Ekiti
}

\begin{abstract}
The importance of physics in human endeavour cannot be glossed over, for it places a vital role and essential part of all human endeavour, especially in science and technology. The study was designed to see the relative and interraction effects of errors in physics practical in Nigeria Secondary Schools. A Quasi experimental design of the three group pre-test, post-test, control design was employed. The sample for the study consisted of sixty (60) students from three selected secondary schools in Nigeria. Equal male and female students were selected using stratified random sampling technique. Physics Practical Questions (PPQ) were validated and used before and after treatment in the groups. The findings revealed that when students are exposed to the idea of errors in practical physics, the degree of accuracy will increase and therefore enhance their performance in the subject. Physics and related courses had been recommended for both male and female students in secondary schools, since sex is not the major issue in physics practical works. If the students are taught how to get accurate results and errors are minimised in physics practical, this will enhance good performance in the subject.
\end{abstract}

Key Words: Physics, practical, laboratory, error, uncertainty, accuracy

\section{Introduction}

Measurement in Physics experiment is limited to certain degree of accuracy due to some errors that normally occur during practical works. Even when valid and reliable apparatus are used. While measuring the potential difference between two points in a conductor, part of the current in the coordinator will always pass through the voltmeter (instrument), thus reducing the original current in the conductor. In this case, what the voltmeter finally registers is slightly different from what it was originally set out to measure.

If errors are too many in measurement, all the efforts, time and money put into it will be waisted noting. For example the effect of "Time Dilation" in the postulate of special relativity as described by Alonso and Finn (1980), is how relative motion affects measurement of time intervals. A clock moving with respect to an observer appears to tick less rapidly than it does when at rest with respect to him.

There is always some degree of errors or uncertainty introduced in our measurement by the very process of measurement. Such errors are not usually important at the microscopic level because of the large magnitude of the quantities being measured. However, at the microscopic level, they are extremely important and play a significant role in any attempt to determine the probable location of the electron in the vicinity of the nucleus.
This study referred to a German physicist who postulated a theory called a methematical model of the atom, which showed that it is not possible to measure both the position and the momentum of subatomic particles simultaneously. This statement is one way of expressing the general principle known as Heinsenberg's uncertainty principle; which state that:

There is always an uncertainty involved in any attempt to measure the position and momentum of the electron simultaneously" which means it is impossible to know simultaneously and with exactness both the position and the Momentum of a particle.

This must be so for the following reasons: To locate the position of the electron, we must shoot another particles at it, such as a photon or another electron, which is the smallest particle we can use. This shows that we are trying to measure the position of a particle with an equal or larger particle; therefore a sizeable error must be introduced in the result. Therefore the uncertainty principle is an indication of the limited of physical measurement and of the limitation of the extent to which we "know" the "true" structure of matter. This principle always serves as a reminder of the limit of all measurement. Another experiment which indicates the impossibility of observing a particle without disturbing it is the case in which we try to determine the position of an electron by means of a microscope. The Xcomponent of momentum of the scattered photon ( $\mathrm{P}$ photon $=\mathrm{h} / \lambda$ ) and the angle $\alpha$, in which it moves has 
an uncertainty. $\Delta \mathrm{p} \sim \mathrm{P}$ photon $\operatorname{Sin} \alpha \mathrm{hd} / 2 \lambda \mathrm{y}$, the uncertainty therefore is a direct consequence of the process of measurement. At the atomic level, measurement inevitably introduces a significant perturbation in the system, due to the interaction between the measuring device and measured quantity. In setting errors the uncertainty or errors may occur in some experiments when a setting is made. In a lens experiment for example, there is an uncertainty in deciding when image of crosswires on a screen is in sharpest focus. The uncertainty or error here can be found by moving the screen until the image is just blurred and noting the distance from this position to that when the image was in sharpest focus. The metre bridge and potentiometer experiments also depend on finding a balance point; the uncertainty or error here may be found by moving the jockey or slider until the galvanometer pointer is just deflected and the distance from this position to the "best" position of balance is noted.

It is therefore necessary to know what causes uncertainty or error in measurement the sources and percentage of a particular error in practical physics. Since it is almost impossible to get 'absolute' value in measurement, the knowledge of percentage error will help in giving a tolerant to remove confusion in the final result.

A number of theories have been postulated on different types of errors in physical science this include: Random, Blunder, Accidental, Systematic, Graphic, Estimated, Instrumental and Environmental errors.

\section{Random Errors}

Random Errors which hold that a random variable do appear on several possible values associated with a probability of number of chances for success divided by the total number of chances or as the limit value to which the relative frequency of occurrence tends as the number of repetitions is increased indefinitely. Raymond (2011), maintained that random errors are caused by intrinsic fluctuations in the apparatus. A simple frequency distribution results from combining two independent random errors. They may arise from ambiguities or uncertainties in the process of measurement, or from fluctuation which are too irregular or fast to be observed in detail. The covariance expresses the mutual interaction between any two random variables. This is expressed in correlation coefficient as

$$
\rho_{x y}=\sigma_{x y}=E\left(\frac{x-\mu_{x}}{\sigma_{x}}\right)\left(\frac{y-\mu_{y}}{\sigma_{x}}\right)
$$

Where $\sigma_{\mathrm{x}}$ and $\sigma_{\mathrm{y}}$ are the standard deviation of $\mathrm{x}$ and $\mathrm{y}$ respectively.

The essential point about random errors is that with the apparatus as it stands no particular random error can be predicted or connected but the sources of random errors can be eliminate.

\section{Systematic Errors}

Error may arise from faults or changes in conditions which could be corrected, such as zero error of an instrument (Barford 2007). In this case the standard or true value must be found. In this case, accurate results will attract subtracting the zero error either form each measurement or from the average measured values. But when 'error' is removed from a measurement the value of that measurement should improve. This idea is applicable to systematic error since the effect occur according to a system which, if known can always be expressed by mathematical formulation $\mathrm{X}_{\mathrm{c}}=\mathrm{X}_{ \pm} \mathrm{e}_{\mathrm{s}}$. They follow a definite pattern, and if the experiment is repeated while maintaining the same conditions, the same pattern will be duplicated and the systematic errors will reoccur.

Raymond (2011) maintained that this depend on the observer, the instrument used or physical environmental conditions of the observational experiment.

There is also accidental error which postulated that a determination of a quantity is the different between the "true value" (which cannot be found) and a determination of the value that is free from blunder or systematic error. The theory of Graphical Error holds that the probability of the occurrence of an error within any range is equal to the sum of the probability of all of the groups within that range and also, if the sice of a single group is used as ordinate, the area under any section of any one of these curves is equal to probability of an error falling within that range. The equation of the curve, which may be derived is written as

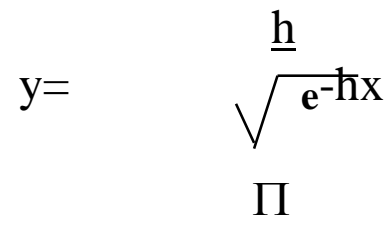

where $\mathrm{e}=$ base of natural logarithms and $\mathrm{x}=$ size of the error

Allan, Hollewed \& Maynes (2000) observed that the true value of a quantity will never be found; or if 
it is found we will never know that we have found it. This argument applies to a single observed quantity only, for example, acceleration due to gravity is known to be about $9.8 \mathrm{~m} / \mathrm{s}$. Like true value, the true errors of a single observed quantity can never be found (Allan, Hollewed \& Maynes, 1980).

If it is possible to determine the amount of error in each examinee's score, it is possible to calculate the standard deviation of this error score of the group. The standard error of measurement can be used to define confidence interval for the students' true scores based on their obtained scores. It also tells us what percentage of the standard deviation is attributable to error of measurement. Since 'true value' is constant, error score then depends on accuracy of the experiment. We can conclude therefore that if observed values are very close to true value, the degree of accuracy of the measurement is very high.

Accuracy in physics practical works plays some vital roles in laboratory work which cannot be glossed over. It is very important to pay attention to value accuracy in science practical because of the negative effect of errors on the outcome of experimental work. It is very important to be more accurate in our practical works because too many errors can render the whole experiment useless. The accuracy should be ensured not only in measurement and observation but in all aspect of the practical up to the calculation and final conclusion. Accuracy must be ensured in measurement, observation, estimation, approximation, calculation interpretation and conclusion.

In measurement Owolabi and Bandele (2009) suggested that, observations should be clearly organised and executed as to be sure that no single error may creep in. He further stated that it is possible to break down gross errors by conducting control measurement. The more accurately the measurement are made, the fewer the errors. A single mistake can lead to many errors and if errors are too many in a single experiment it will render the whole exercise useless.

\section{Hypotheses}

Two null hypotheses (Ho) were raised for this study: Ho$_{1}$ : There is no significant difference between the relative effect of ECIP and conventional method on errors committed in physics practical.

$\mathbf{H o}_{\mathbf{1}}$ : There is no significant interraction effect of sex and type of treatment on errors committed in physics practical.

\section{Design}

A Quasi experimental design was employed for the study, using a pre-test, post-test three group design. The experimental group (X) was exposed to treatment, using "Error Correcting Instructional Package (ECIP) which was the researcher's special design to minimise errors in physics practical. The conventional group $(\mathrm{Y})$ were exposed to the normal physics conventional practical approach, while the control group were left without any special method. The design of the study is as follows:

\begin{tabular}{|c|c|}
\hline Experimental group (X) & $\mathrm{O}_{1} \quad \mathrm{X} \quad \mathrm{O}_{2}$ \\
\hline entional group (Y & $\mathrm{O}_{3}$ \\
\hline ontrol group & $\mathrm{O}_{5}$ \\
\hline
\end{tabular}

\section{Sample and Sampling Technique}

The sample for this study consisted of sixty (60) students from three selected State Government Colleges in Nigeria. In each school selected from different Local Government headquarter, twenty (20) students were used. Equal male and female students were selected using stratified 20 item Physics Practical Test (PPT) was used to selected the students used random sampling technique. The students in the three schools were considered to be of comparable academic standard.

\section{Instrumentation}

The instruments used for the study include: Physics Practical Test (PPT) and Physics Practical Questions (PPQ). The research employed the use of a 20-item Physics Practical Test to ensure the homogeneity of the students. The Physics Practical Question (PPQ) which were extracted from West African Examination Council (WAEC) past questions was also employed to know the student level of errors committed/accuracy in physics practical works before and after treatment. Four common areas in physics practical were also employed which include: Mechanics, Heat, Light and Electricity. Marking guides prepared by the WAEC each experiment were used for marking and ranking of errors in students' reports.

A special package designed by the researcher called Error Correcting Instructional Package (ECIP) was used for treatment in experimental group (X). the package was designed in form of classroom instructional package with emphasis activities to be 
performing by students and the easiest way for the teachers to used questioning, laboratory techniques and demonstration methods with objective of exposing students to skills for minimizing errors in Physics Practical works. The split-half reliability of PPQ using pearson moment correction coefficient was 0.65 and supported with full length using Spearman Brown prophesy formula to be 0.79 .

\section{Procedure and Data Collection}

The students selected from the schools were exposed to Physics Practical experiments in Mechanics, Heat, Light and Electricity at the pre-test stage. The ECIP was applied as treatment to the first group (X) for six consecutive weeks after the pre-test while the normal conventioned method was used for the second group (Y) and the third group (Z) which serves as control group were left untreated with any special method. The two groups $\mathrm{X}$ and $\mathrm{Y}$ were taught by well trained qualified and experienced physics teachers. The teacher used for group $\mathrm{X}$ was trained how to use
ECIP while teacher in group $\mathrm{Y}$ was allowed to use normal conventional method.

The errors committed were ranked and collated at each stage of every experiment. The ten common identified errors in Physics Practical works were collated from students reports. The performance of students were analysed using descriptive statistics such as mean and standard deviation. The two hypotheses raised were tested using inferential statistics t-test and analysis of covariance (ANCOVA) respectively. The results were upheld at 0.05 level of significant.

HO$_{\mathrm{I}}$ : There is no significant difference between the relative effects of ECIP and conventional method on errors committed in physics practical.

To calculate the relative effects of the ECIP and conventional approach on errors committed in physics practical, errors scores in the experimental and conventional groups were subtracted from that of the control group, (Z-X and Z-Y) respectively. These relative lie scores were then compared. The hypothesis was tested using t-test at 0.05 level of significant.

Table 1. t-test summary on relative effects of treatment and conventional methods.

\begin{tabular}{|c|c|c|c|c|c|c|}
\hline Groups & & $\mathrm{N}$ & $\overline{\mathrm{X}}$ & S.D & $\mathrm{t}$-cal & t-table \\
\hline Relative ECIP score (Z-X) & & 20 & 14.25 & 7.68 & 5.78 & 2.093 \\
\hline Relative Conventional Score & $(\mathrm{Z}-\mathrm{Y})$ & 20 & 3.25 & 7.41 & & \\
\hline
\end{tabular}

Since the t-calculated value 5.25 was higher than the t-table value 2.093, at 0.05 level of significance, the null hypothesis was rejected. There was a significant difference between the relative means of two groups. Hence the relative effect of ECIP and conventional approach was significant on errors committed in physics practical. The mean values of 14.25 and 3.25 for relative effect in treatment and conventional group respectively revealed positive influence of the ECIP on student achievement in practical works. As a result of using ECIP, students committed fewer errors in physics practical works. Figure 1 and 2 give graphical presentation showing the position of the influence of ECIP on treatment group over conventional group. 
Pre-Test Error Scores in Groups $1 \& 2$

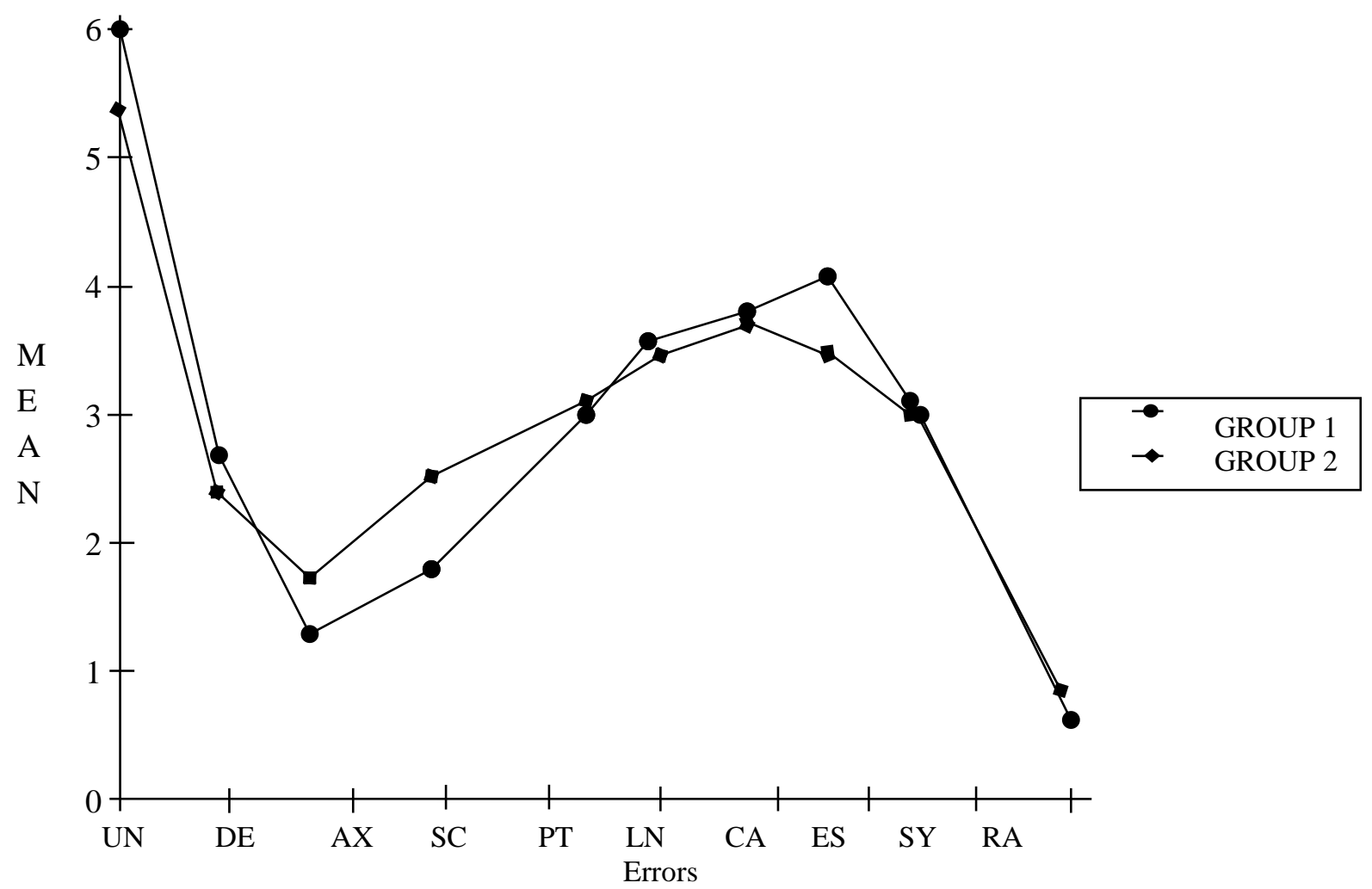

Figure 1 revealed that errors committed by the students in the two groups were nearly the same. Which means there were no difference in the performance of treatment and conventional groups in their pre-test experimental works.

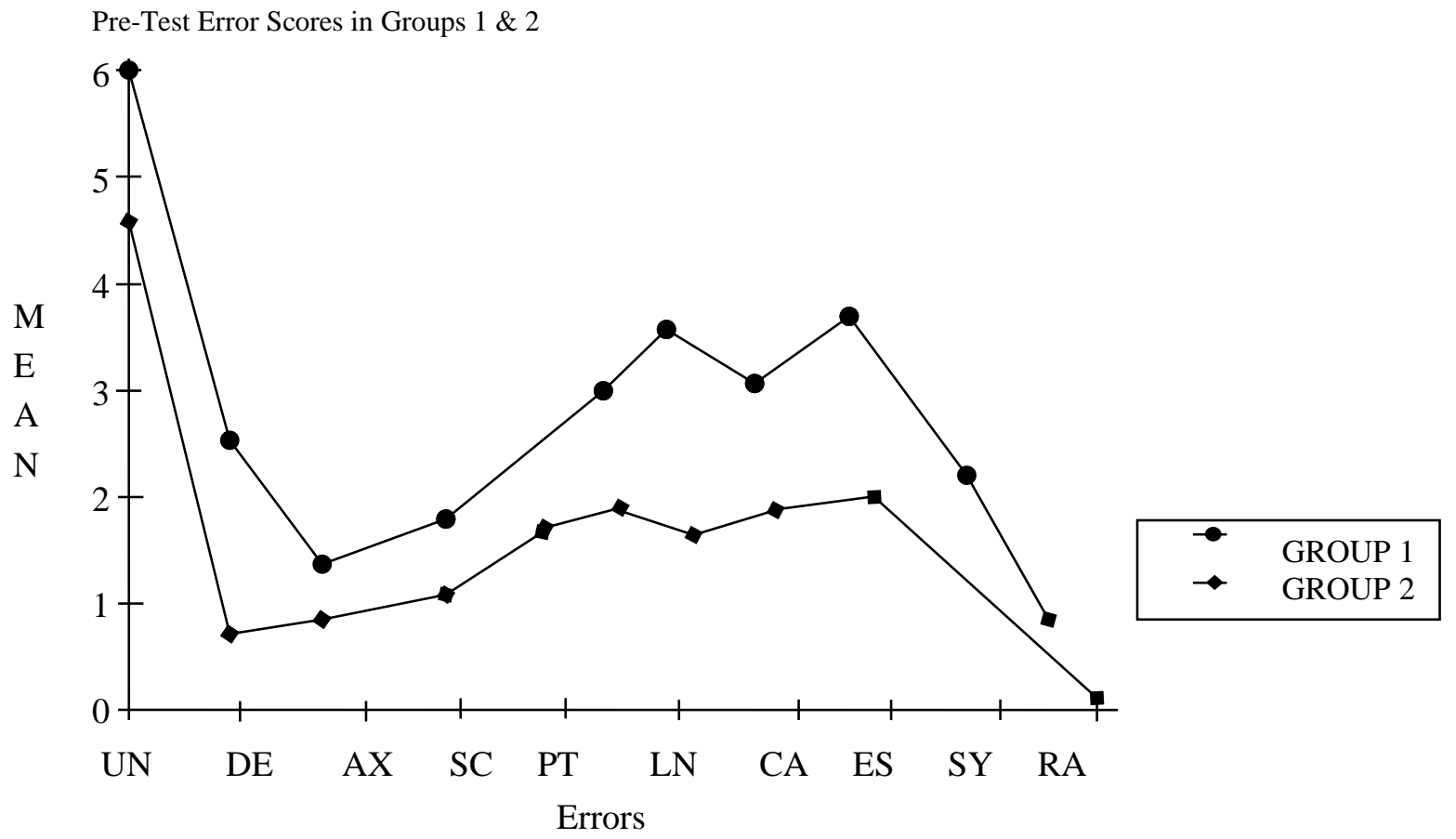

Figure 2 revealed the effect of ECIP on treatment over conventional group. 
All the errors were drastically reduced after treatment with ECIP when compared with conventional group. The improvement in the use of ECIP confirms its effectiveness on errors committed during students' practical works.
$\mathrm{Ho}_{2}$ : There is no significant interaction effect of sex and type of treatment on errors committed in physics practical. The hypothesis was tested using Analysis of Covariance (ANCOVA). The results are presented in tables 2 and 3 .

Table 2. Summary of ANCOVA on the post-test mean errors over treatment and conventional group with sex.

\begin{tabular}{lrrrrr}
\hline Source of variation & \multicolumn{1}{c}{ SS } & Df & M.S & F & P \\
\hline Covariate PRTST & 514.029 & 1 & 514.029 & 9.127 & 0.05 \\
& 514.029 & 1 & 514.029 & 9.127 & 0.05 \\
Main Effect Sex & 1219.422 & 2 & 609.711 & 10.825 & 0.000 \\
Group & 201.498 & 1 & 201.498 & 3.578 & 0.067 \\
& 957.170 & 1 & 957.170 & 16.995 & 0.000 \\
2-way interaction & & & & & 0.065 \\
Sex X group & 3.651 & 1 & 3.651 & 0.065 & 0.801 \\
& 3.651 & & 3.651 & 7.711 & 0.000 \\
Explained & 1737.102 & 4 & 434.275 & & \\
Residual & 1971.273 & 35 & 56.322 & & \\
Total & 3708.375 & 39 & 95.087 & & \\
\hline
\end{tabular}

Table 2 shows significant main effects for the groups $(\mathrm{F}=16.995 ; \mathrm{P} \leq 0.05)$ but not for the sexes $(\mathrm{F}=0.065$; $\mathrm{P}<0.80)$. In order to provide some indications for the errors committed by the two groups (treatment and conventional groups), a multiple classification Analysis (MCA) was computed. The results are presented in Table 3.

Table 3. Multiple classification of post-test mean error of treatment and conventional groups with sex.

\begin{tabular}{|c|c|c|c|c|c|}
\hline Variable + Category & $\mathrm{N}$ & $\begin{array}{l}\text { Unadjusted } \\
\text { Deviation }\end{array}$ & Eta & $\begin{array}{l}\text { Adjusted for dependent }+ \\
\text { covariate Deviation }\end{array}$ & Beta \\
\hline \multicolumn{6}{|l|}{ Sex: } \\
\hline Male & 20 & -3.04 & & -2.27 & \\
\hline \multirow[t]{2}{*}{ Female } & 20 & 3.02 & & -2.27 & \\
\hline & & & 0.31 & & 0.24 \\
\hline \multicolumn{6}{|l|}{ Group } \\
\hline Experimental & 20 & 3.08 & & 5.40 & \\
\hline conventional & 20 & -3.08 & & -5.40 & \\
\hline & & & 0.32 & & 0.56 \\
\hline Multiple $\mathrm{R}^{2}$ & & & & & 0.467 \\
\hline Multiple R & & & & & 0.684 \\
\hline
\end{tabular}

Grand Means $=44.875$

The MCA (table 3) shows that the male has a very close adjusted mean (22.60) on errors committed in groups for post-test, compared with that of the female (22.56) hence, not significantly different. It also shows that the conventional group $\left(\mathrm{G}_{2}\right)$ has the higher adjusted mean between the two groups, while treatment group has the lower adjusted mean (14.40).

On the bases of these findings hypothesis 2 was therefore not rejected. There was no significant interaction effect of sex and type of treatment on errors committed in physics practical.
The performances of male and female sexes were marginal. Therefore sex is not a major issue as far as committance of errors in physics practical is concern. The level of errors committed by male students in physics experiment was found proportional to their female counterpart.

\section{Discussion}

Hypothesis 1 sought to find the relative effect of ECIP and conventional approach on errors committed 
in physics practical. This study has also revealed that the relative effects of ECIP and conventional approach were found significantly different on errors committed in physics practical. The use of ECIP on treatment group and conventional approach in group 2 has different impact on their achievement. Even though there was improvement in the two groups (treatment and conventional) but the findings revealed that treatment groups committed less errors in all the experiments. The mean error 14.25 in treatment group compared with 3.25 in conventional group showed that errors were appreciably reduced after the use of ECIP. The comparism of the two groups in the pre-test and remarkable improvement in the post-test revealed that errors cannot but occur in physics practical work, but can be reduced to the minimum by using ECIP. To have a sound base on which to build practical techniques for the design and analysis of experiment, some elementary understanding of experimental errors and associated probability theory is essential (Bannister and Raymond 2008).

The ECIP was found very effective for reducing errors in physics practical works as we could see most of the errors being reduced to the minimum some of these are Decimal, Axis, Scale, Random and many others. The presence of the remaining errors in the entire post-test treatment group confirmed the study of Allan, Hollewed and Maynes, (2000). They observed that the true value of a quantity will never be found; or if it is found we will never know that we have found it. The objective in any physics practical experiment should be to endeavour to reduce the errors to the minimum.

Hypothesis 2 sought to find the interaction effect of sex and type of treatment on errors committed in physics practical. The findings revealed that there was no significant difference in performance between male and female students in the experimental groups. The findings showed that sexes of students have no effect on their performance or effectiveness in laboratory works. The errors committed by male students were almost the same with those committed by their female counterparts. This was supported by Daramola in Owolabi (2009) in their studies. According to them both sexes received encouragements to use their intellectual gift without sex discrimination. Since the gifted girls do not differ from gifted boys.

\section{Conclusion and Recommendations}

The importance of physics in human endeavour cannot be glossed over, for it plays a vital role and essential part of the intellectual life of man. Physics has been regarded as a necessary part of all human endeavour. Physics practical especially plays a significant role in national building, particularly in Science and Technology.

Errors cannot but occur in physics practical, but the outcome of good experimental work depends on accuracy of the work. If 'observed values'are very close to "true value" the degree of accuracy of the measurement will be very high. This can only be experienced by avoiding errors as much as possible. Physics students should therefore be exposed to the idea of errors, how to calculate percentage error, the danger of too many errors in practical works and how to reduce the errors to the minimum.

Students are always motivated when their works in laboratory yeild good results. Since Error Correcting Instructional Package (ECIP) has content that is considered adequate and appropriate for error reduction it will be appropriate for secondary school syllabus and practical text books. This will enhance good performance, as students will be taught how to get accurate results in physics practical works. The inculcation of Error Correcting Instructional Package (ECIP) content in the syllabus therefore could serve as reinforcement to teaching and learning of physics at all levels of science education globally.

Since sex is not the major issue in physics practical works, as male and female performed equally in their practical (experimental) works. In choosing of carrier therefore female students should be encouraged to offer physics in high schools and tertiary institutions. Writers of physics practical text books and instructors' guides should endeavour to list in clear terms at the end of each topic treated the errors that students are likely to commit and necessary steps to avoid them.

\section{References}

Allan, AL; Hollewey J. R \& Maynes J.H.B. (2000): Practical Surveying and Computation, Heinemann Publisher London 200-249.

Alonso M. \& Finn E. J. (2010): Fundamental University Physics (III) Quantitative and Statistical Physics Addison-Westley Publishing Company Inc. (reversed edition).

Bannister A \& Raymonds (2008): Surveying the English Language Book Society and Pitman London 590-625.

Barford N. C. (2007). Experimental Measurements Precision, Error and Truth. Addison-Wesley Publishing company, Inc London 72-129.

Raymond E. D. F. (2011): Surveying Theory and Practice. Mc Graw Hill Publisher Company New York.

Owolabi O. T. \& Bandele S. O. (2009): Effect of Error Correcting Instructional Package (ECIP) on Physics Practicl Lessons in Nigeria Secondary Schools. International Journal of Science Education, Bogota Colombia. 10(1),47-49. 